\title{
Evaluation of the Disaster-related Information Process from the Perspective of Managers in the Iranian Red Crescent Society
}

\section{Arezoo Ghasemi Siani ${ }^{1} \mathbb{D}$, Mohammad Reza Alibeik $^{2} \mathbb{D}$, Hesam Seyedin ${ }^{3} \mathbb{D}$, Milad Ahmadi Marzaleh ${ }^{4,5,6,8} \mathbb{D}$, Ahmad Soltani ${ }^{7,8} \mathbb{D}$}

Date of submission: 18 Aug. 2020

Date of acceptance: 16 Dec. 2020

\section{Original Article}

\begin{abstract}
INTRODUCTION: Information is known as the main source of power over others. In this respect, the use of correct, accurate, and timely information in decision-making, planning, and other related issues can be effective in the outcome of activities and services provided by organizations. In times of crisis and disaster incidence, information plays a key and crucial role and affects various disaster response processes. This study was conducted to investigate the communication and information processes and information analysis in the Iranian Red Crescent Society at the time of disasters in 2019.

METHODS: This qualitative study with a content analysis design was carried out on 17 experts and managers in the Iranian Red Crescent Society. The necessary data regarding the existing information processes were collected through performing interviews with the participants. The collected data from conducting and implementing the interviews were then coded in MAXQDA software using qualitative analysis method and subjected to analysis.

FINDINGS: Based on the findings, the majority of the activities of this organization were carried out by holding meetings to make the necessary arrangements. Measures, such as public education and reports to journalists, media, and social networks were provided in the form of written instructions and protocols. It was revealed that such communication means as media, newspapers, 112 emergency SMS system, and Thuraya satellite network were used for information. The tool utilized in the information process in the Iranian Red Crescent Society was the Disaster Management Information System to record information and the history of crises. This system was located and used in the Control and Coordination Center, and recorded all documents related to the events, including photos, videos, and reports and information of rescuers.

CONCLUSION: The most significant problems that crisis management is consistently confronting are the delays in realizing the dimensions of a crisis, lack of information needed to make decisions, and commencement of recovery activities. In addition, it is highly important to have accurate information to be able to respond properly. The acquisition, storage, retrieval, and analysis of information can be the most prominent factors in the success of crisis management.
\end{abstract}

Keywords: Crisis; Disasters; Information Process; Iran; Red Crescent.

How to cite this article: Ghasemi Siani A, Alibeik MR, Seyedin H, Ahmadi Marzaleh M, Soltani A. Evaluation of the Disaster-related Information Process from the Perspective of Managers in the Iranian Red Crescent Society. Sci J Rescue Relief 2020; 12(4): 235-41.

\footnotetext{
1- MA Student, Department of Library and Medical Information, School of Management and Medical Information, Iran University of Medical Sciences, Tehran, Iran

2- Instructor, Department of Medical Library and Information, School of Management and Medical Information, Iran University of Medical Sciences, Tehran, Iran

3- PhD, Department of Health in Disasters and Emergencies, School of Management and Medical Information, Iran University of Medical Sciences, Tehran, Iran

4- Research Center for Health Management in Mass Gathering, Iranian Red Crescent Society, Tehran, Iran

5- PhD, Health in Disasters and Emergencies, School of Management and Medical Information, Shiraz University of Medical Sciences, Shiraz, Iran

6- MPH in Health Policy, Health Policy Research Center, Shiraz University of Medical Sciences, Fars, Iran

7- Research Center for Emergency and Disaster Resilience, Iranian Red Crescent Society, Tehran, Iran

8- PhD, Applied Science Higher Education Institute, Red Crescent Society of the Islamic Republic of Iran, Tehran, Iran

Correspondence to: Ahmad Soltani, Email: soltani.amd@gmail.com
} 


\section{Introduction}

I nformation and communication technology is one of the tools connecting organizations and society, and accurate and timely information is considered one of the key activities in any organization. Information is known as the main source of power over others. In this respect, the use of correct, accurate, and timely information in decision-making, planning, and other related issues can be effective in the outcome of activities and services provided by organizations (1). Moreover, the application of the right information processes would save a lot of money, manpower, and service provision at the right time and place, as well as providing the necessary preparations and facilities. Therefore, in organizations that are in charge of providing services, one of the basic needs is an effective and efficient information system.

Crises are the causes of irreparable personal, social, and national losses and damages that include simple injuries and disabilities to economic, social, and political crises. The prediction of the necessary measures to prevent, prepare for, and respond to crises and disasters to reduce vulnerability against such circumstances will decrease their negative consequences (3). There are various challenges related to the duties and roles of relief agencies, which parallel work and unclear duties and roles of each of these agencies in providing specialized services and issues related to the process of informing about the required services in time of crisis incidence are among these challenges and can make the proper function of organizations difficult (4).

The Iranian Red Crescent Society is one of the largest relief organizations in the country responsible for assisting people affected by natural and in some cases man-made disasters in the country (2). Consequently, it requires to have appropriate and coherent programs to deal with the crisis in all dimensions and play an important role in timely relief and reduction of casualties by evaluating and improving information processes in its extensive network throughout the country. Based on the existing experiences of crisis and disasters that occurred in Iran over the past years, it can be realized that one of the effective factors in crisis prevention will be prompt and timely information. National-coordinated early warning systems based on the climatic, geographical, and urban areas information are among the essentials of the country's rescue network.
The identification and elimination of existing barriers in the field of human resources, equipment, training, and accountability can be accomplished by removing overlaps, developing disaster response plans, designing and possessing an efficient national information system equipped with an alert system, and improving the process of crisis incident informing will improve the performance of the Red Crescent population. On the other hand, the level of productivity and quality of operations is boosted by employing the timely and integrated information process. In this respect, with timely and accurate information, rescue operations, first aid, and medical and health interventions that are directly related to human health and life will be performed with less difficulty (5).

Detailed research was carried out on English and Persian databases; however, no specific research has been performed in this domain. Therefore, this study was conducted to investigate the managers' attitudes to the disaster-related information process in the Iranian Red Crescent Society.

\section{Methods}

This qualitative study was conducted based on an inductive content analysis approach in 2019. The required information was collected through interviews with relevant experts and managers. The study population included experts and managers present in the Deputy Secretary of Health, Treatment, and Rehabilitation, Rescue and Relief Organization, Deputy of Education, and the headquarters of the Iranian Red Crescent Society. The inclusion criteria were having at least a bachelor's degree, work experience in disaster and crisis management, and at least 2-year work experience, as well as a willingness to participate in research and collaboration.

The interviews were conducted using openended questions with the experts, and they were informed about the possibility of study withdrawal at any research stage. The interview guide was first compiled by reviewing texts and documents related to the objectives of the research, followed by reviewing, evaluating, summarizing, and correcting the questions by the experts. The final interview form was designed containing openended and demographic questions (Table 1).

The samples were selected using purposive and snowball sampling methods. The eligible cases were identified and explained about the goals and importance of the research. After 
Table 1. Open-ended questions

1. How do the information centers of the Red Crescent Society and related organizations, such as Crisis Management and Ministry of Health and Medical Education interact with each other?

2. Do you think that crisis-related information to the responsible units has a specific protocol with a standard procedure that does not cause rework between the Red Crescent Society and other units, such as crisis management?

3. What measures have been adopted regarding informing the community members using mass media, such as telephone, fax, radio, television, and the respondent system?

4. Do you think that the Red Crescent Society has employed specialized, trained, and interested personnel in the field of communication and information to provide correct, desirable, and timely information to the responsible units?

5. Today, the pace of information is the main element that is important in organizations, especially in relation to rescue and relief services. To what extent has the Red Crescent Society paid attention to this matter and improved it?

6. What strategies should be considered to improve the process of informing the rescue services to the responsible units regarding how to deal with the crisis?

7. What infrastructures have been considered for the Red Crescent Society in relation to improving the information process, such as increasing the speed, accuracy, and precision of information; employing specialized forces; and allocating financial resources?

8. How are the crises and crisis-related rescue operations recorded? What instruments are used in the information process?

9. In your opinion, have the main applicants and beneficiaries of information and the units involved in rescue affairs failed to receive information due to the lack of defined and clear communication with information centers? If yes, what do you think is the reason?

10. What are the strengths and weaknesses of crisis-related information processes among responsible organizations?

obtaining their consent, the researcher interviewed them at the appropriate time. Numbers rather than the subjects' names were included in the demographic forms. Interviews were continued until reaching theoretical saturation, which all were conducted by the same researcher.

Notes were taken during the interviews and the interviews were recorded to avoid possible problems, which were applied immediately after the interview. If necessary, the interviews were made available to individuals to modify or add to them. The collected data were analyzed using the thematic analysis method to extract information processes in the Iranian Red Crescent Society.

Thematic analysis is a six-step process performed as follows: a) Familiarization with the data, b) Creating primary codes, c) Searching for themes, d) Reviewing themes and subthemes, e) Defining and naming themes and subthemes, and) Writing up the report (6). The four-dimension criteria proposed by Lincoln and Guba, namely credibility, transferability, dependability, and confirmability, were implemented to improve the validity and reliability of the qualitative study. Finally, the interview replies were analyzed, coded, and categorized in MAXQDA software and the categories and subcategories were extracted.

\section{Findings}

Interviews were conducted with 17 experts and specialized managers at the Iranian Red Crescent Society and continued until reaching theoretical saturation. Each interview lasted an average of 45 min. All participants were male with a mean age of 48.15 years and 17-year work experience. Demographic and occupational information of the participants is presented in Table 2. After analyzing the content of the interviews, 215 codes were finally extracted, which by deleting

Table 2. Academic and occupational information of the research participants

\begin{tabular}{|cccc|}
\hline Variable & & Number & Percentage \\
Rank & Bachelor & 4 & 23.5 \\
& Masters & 8 & 47 \\
& PhD & 5 & 29.5 \\
\multirow{4}{*}{ Position } & General manager & 4 & 23.5 \\
& Head of the office & 8 & 47 \\
& Expert & 5 & 29.5 \\
\hline
\end{tabular}


Table 3. Themes and subthemes of the interaction processes at information centers in crisis

Theme

Theme
Through the working groups
of the Crisis Headquarters

information centers

Internal coordination

\section{Subcategory}

Holding meetings for coordination

Send default SMS in times of crisis

In the form of action within specific frameworks

Written protocols for times of crisis Coordination through the emergency operations center Thuraya satellite network

Table 4. Categories and subcategories of the theme related to the process of informing society members in crisis

\begin{tabular}{|c|c|c|}
\hline Theme & Category & Subcategory \\
\hline \multirow[t]{2}{*}{ Process of informing society members } & Mass media & $\begin{array}{l}\text { public education } \\
\text { Report to journalists } \\
\text { Newspapers and radio } \\
\text { SMS system } 112\end{array}$ \\
\hline & Social networks & $\begin{array}{c}\text { Red Crescent Society Website } \\
\text { Telegram and Instagram }\end{array}$ \\
\hline
\end{tabular}

duplicate codes and re-analyzing the remaining codes, were classified into five themes. Tables 3-7 summarize these themes with identified categories and subcategories.

After analyzing the interviews regarding the interaction of information centers in the crisis incidence, two categories and six subcategories were extracted (Table 3).

After analyzing the interviews about the process of informing society members in crisis incidence, two categories and six subcategories were extracted (Table 4).

After analyzing the interviews regarding the information standard in the crisis, two categories and four sub-categories were extracted (Table 5).
After analyzing the interviews regarding the strategies for improving the information process in the crisis, two categories, and five subcategories were extracted (Table 6.)

After analyzing the interviews regarding the weaknesses of the information process in the crisis, two categories and six subcategories were extracted (Table 7).

As is shown in tables 3 to 7 , the basic categories were identified to examine the current state of the crisis-related information process in the Iranian Red Crescent Society. These categories alone cannot carry a special meaning to investigate the current state of the crisis-related information process in the Iranian Red Crescent

Table 5. Categories and sub-categories of the theme related to the standard information procedure in crisis

\begin{tabular}{c|cc}
\hline \multicolumn{1}{c|}{ Theme } & Category & Subcategory \\
& Internal information & $\begin{array}{c}\text { SMS to senior executives } \\
\text { Emergency plans }\end{array}$ \\
Standard information procedure & & $\begin{array}{c}\text { Recall of forces } \\
\end{array}$ \\
& External information & \begin{tabular}{c} 
Instructions for forming the national headquarters \\
\hline
\end{tabular} \\
\hline
\end{tabular}

Table 6. Categories and sub-categories of the themes related to the strategies for improving the information process in

\begin{tabular}{|c|c|c|}
\hline Theme & Category & Subcategory \\
\hline \multirow{2}{*}{$\begin{array}{l}\text { Strategies for improving } \\
\text { the information process }\end{array}$} & $\begin{array}{l}\text { Access to an appropriate } \\
\text { information platform }\end{array}$ & $\begin{array}{l}\text { Upgrade system equipment } \\
\text { More cooperation with the media } \\
\text { Use advanced mobile tools }\end{array}$ \\
\hline & Update public relations guidelines & $\begin{array}{l}\text { Develop a manual in accordance with } \\
\text { international standards } \\
\text { Training in line with the needs of the day }\end{array}$ \\
\hline
\end{tabular}


Table 7. Categories and subclasses of the themes related to the weaknesses of the information process in crisis

\begin{tabular}{ccc}
\hline Theme & Category & Subcategory \\
weaknesses of the & Lack of infrastructure \\
information process & Intra-organizational factors & $\begin{array}{c}\text { Lack of alternative protocols for social networks } \\
\text { Lack of high coordination between departments } \\
\text { Need to strengthen education in the field of information } \\
\text { and public relations }\end{array}$ \\
& External organizational factors & $\begin{array}{c}\text { Need for crisis managers to work collaboratively } \\
\text { Lack of strong inter-organizational interaction }\end{array}$ \\
\hline
\end{tabular}

Society. In the section on how to communicate with information centers, it was determined that the activities were carried out in the form of meetings to make the necessary arrangements. These activities were performed in the form of frameworks written by the Crisis Management and Red Crescent managers, and instructions available in the Crisis Management Headquarters are given to the specialized working groups. Based on the results, both the Thuraya satellite network and written protocols for crisis times in the emergency operations center (EOC) were used in the intra-organizational information process of the Red Crescent Society.

In the field of standard information procedure, such measures as public education, reporting to journalists, SMS systems, and social media and networks were considered in the form of written instructions and protocols. Among mass media, such tools as media, newspapers, 112 emergency SMS system, Thuraya mobile phone, and reporting to journalists were employed. It was found that most of the information personnel working in the information field of the Red Crescent Society were expert, experienced, and interested in the domain of communication and public relations.

The items that were obtained to improve the information speed of rescue services included the existence of instruction handbooks in the information process and the employment of specialized and professional individuals in the information-related affairs. Moreover, such strategies as strengthening and equipping information systems, considering a single spokesperson in times of crisis, strengthening and updating existing public relations guidelines, and accessing an appropriate information platform by strengthening tools, as well as media cooperation with the Red Crescent Society, were provided to improve the information speed of rescue and relief services.
The tools available in the information process of the Red Crescent Society utilize the disaster management information system (DMIS) to record information and history of crises, which is conducted in the Control and Coordination Center. This system records all the documentation related to the events, including photos, videos, and reports, and the information of the rescuers. In addition, the Red Crescent Society has a library and a documentation center. The Red Crescent Society has not faced a problem in recent years due to the strengthening of its information infrastructures and rapid receiving of incident information through the EOC. However, it was found to be parallel work and disruption in the field of joint activities with the working groups of the Crisis Staff.

The most frequent strengths of information processes were reported to be the improvement of the promptitude in information, increase in cooperation with provincial deputies, more interaction with the media, improvement of using advanced information tools technology. However, some weaknesses were also observed that included lack of strong inter-organizational interactions, lack of a single spokesperson, and lack of coordination between departments, as well as the strengthening of existing training in the field of information.

\section{Discussion and Conclusion}

The information process of the rescue and relief services provided by the Red Crescent Society uses crisis registration systems in the Control and Coordination Center of Red Crescent Society, DMIS system, media and social networks in times of crisis incidence, satellite technologies, emergency telephone, and Thuraya mobile system. Zaboli et al., in their study, reported the existence of an early warning system and standardization of the indicators of this system to be able to deal with the crisis. An early warning 
system can detect clear disasters and signals in the affected communities (7).

Rezaie mentioned the cooperation of media and news sites with the Red Crescent Society and emphasized the need for the existence of active media centers for timely information and more support and cooperation of organizations involved in rescue and relief (8). In their study, Kinsel et al. highlighted the necessity of a crisis management system by introducing the crisis and its threatening dangers in emergencies and presented the content management system for information to facilitate communication. This system manages resources and provides access to crisis information to authorized users of the content management system (9). Activities are implemented in the form of frameworks developed by specialized crisis management working groups at the EOC Center with existing protocols. Written instructions available at the Crisis Management Headquarters, which are delegated to the Crisis Management Working Groups through numerous meetings, have been strengthened through public education and the creation of public relations guidelines in the form of an information services booklet.

Mirmaradzehi, in his study, referred to the need for interaction between the Red Crescent Society and crisis management managers in times of danger and showed that information technology and the existence of media requirements effectively reduced the damage caused by natural and man-made disasters (10). In a study carried out by the International Committee of the Red Cross, it emphasized the necessity of effective communication facilities and an efficient and timely information system. Furthermore, it pointed to the existence of effective information and communication systems, the presence of a specialized medical team, health supplies and facilities, and fast-built hospitals regarding the mechanism of health information service standards (11).

It is necessary to strengthen communication infrastructures and information equipment in line with global standards. In the working groups of the Crisis Management Headquarters, there was often disturbance and parallel work in the role and duties of the rescue and relief working group and the Red Crescent Society. There was no strong interaction with the organizations involved in rescue and relief due to the lack of crisis managers' effort to work collaboratively.
Regarding social networks, there was no alternative protocol for information. Therefore, one of the important issues in the field of information process challenges is strengthening existing training in the field of information.

Yousefi introduced information as an important element in reducing vulnerability. He also states that the communication and information equipment available in crisis management was not in a good condition and had a significant difference compared to international standards (12). Leidner et al. carried out a comparative study and reported the need for the employment of information technology and the ability to detect signals. The results of their study showed that the improvement of coordination mechanisms, including organizational, information, and technology response structure were important factors in reducing incoordination in times of crisis (13).

Any critical event requires a major relief effort to minimize its consequent damages. The activity of the relief operations coordinator system will be most effective if it relies on public relations based on a scientific basis (14). The role of public relations and information as the main and trustworthy advisory body of the General Operations Manager, will not only facilitate the executive duties of management but also makes it possible to prevent potential worst consequences in the future, even in the political, social, legal, and economic sectors. The presence of such public relations, alongside the relief operations coordinator system, contributes to sustainable development since the public relations duties start before the occurrence of each crisis and continues for a long time after the end of the relief operation.

It is suggested to adopt the following measures in line with the results of the present study: 1) creating a suitable platform for crisis management, such as communication systems and telecommunication equipment, 2) creating a suitable platform for members of the crisis management team to perform maneuvers or exercises, 3) monitoring the installation of communication equipment in the EOC center and ensuring of the communication channels established with other EOC centers in the provinces, 4) ensuring of the provision of communication equipment, such as radio, telephone, fax, and computer and related services, to the EOC staff as much as needed, 5) providing clear, accurate, and complete news information and 
reducing the occurrence of rumors about the incident by updating the public relations and information department, 6) attracting public opinion on the accuracy of information and news and removing ambiguities by reassuring the public about the reliability of the news source, 7) citing public relations as a reference by managers within the organization and the media and coding and transmitting news in times of crisis aiming to resolve tensions and planning based on accurate information, 8) issuing corrections, announcements, denials, and news approvals timely that are appropriate in terms of setting and expressing the problem, 9) using simple descriptions when reporting an emergency to be understandable for the public and avoiding using specialized and incomprehensible words for non-specialists, 10) performing the informing and demanding role by the media in accordance with the region in each crisis, with the ultimate goal of restoring hope to the people in crises affecting humans and country, and 11) identifying and prioritizing needs by the media through proper, well-defined communicating with the Red Crescent Society, and informing the people honestly and timely to save the country from the crisis situation.

The most significant problems that crisis management is consistently confronting are the delays in realizing the dimensions of a crisis, lack of information needed to make decisions, and commencement of recovery activities. In addition, it is highly important to have accurate information to be able to respond properly. The acquisition, storage, retrieval, and analysis of information can be the most prominent factors in the success of crisis management.

\section{Acknowledgments}

This article is extracted from a master's dissertation entitled "Review of the current status of the crisis-related information process in the Iranian Red Crescent Society" with the code of iums/shmis-97-3-37-12829, supported by the Iran University of Medical Sciences, Tehran, Iran.

\section{Conflict of Interests}

Authors have no conflict of interests.

\section{References}

1. Sadatnia F. Investigating the impact of information systems on improving the decision making of managers of Sari Mellat Bank. [Master Thesis]. Tehran: Islamic Azad University; 2015. [In Persian].

2. Statute of the Red Crescent Society of the Islamic Republic of Iran. Tehran: Iranian Red Crescent Society; 2009.

3. Aghamiri SS, Farsad H, Oveisi N, Kavehfirouz Z, Abjam Z, Mousavi AS, et al. View assessment of the affected people of East Azerbaijan province about Red Crescent society relief operations at earthquake in August 2012. Quart Sci J Rescue Relief 2013; 5(1): 64-55.

4. Mousavi AS, Majidi N, Mousavi SM. Reflection of the Islamic Republic of Iran Red Crescent Society in the official newspapers \& news agencies in winter 2012. Quart Sci J Rescue Relief 2013; 5(3): 45-58.

5. Ministry of Health and Medical Education. Available at: URL: http://www.behdasht.gov.ir/; 2018

6. Braun V, Clarke V. Using thematic analysis in psychology. Qual Res Psychol 2006; 3(2): 77-101.

7. Zaboli R, Seyedin S, Malmoon Z. Early warning system for disasters within health organizations: a mandatory system for developing countries. Health Promot Perspect 2013; 3(2): 261.

8. Rezaei S. A study of the factors influencing on the on time services of search and rescur by Red Crescent Organization. [Master Thesis]. Tehran: Islamic Azad University; 2015. [In Persian].

9. Kienzle J, Guelfi N, Mustafiz S. Crisis management systems: a case study for aspect-oriented modeling. Transactions on aspect-oriented software development. Berlin, Heidelberg: Springer; 2010.

10. Mirmorad Zehi MA. The role of information technology in reducing tsunami damage in Southern Coasts of Iran (Chabahar). [Master Thesis]. Zahedan: The University of Sistan \& Baluchestan; 2012.

11. International Committee of the Red Cross (ICRC). Health care in danger: making the case. Geneva, Switzerland: International Committee of the Red Cross; 2011.

12. Yousefi AS. An investigation and study on information systems for natural crises management in ministry of road \& transportation and propound optimal proposal for its organization. [Master Thesis]. Tehran: Payam Noor University; 2012.

13. Leidner DE, Pan G, L PS. The role of IT in crisis response: lessons from the SARS and Asian Tsunami disasters. Strategic Inform Syst 2009; 18: 80-99.

14. Tian X, Zhao G, Cao D, Wang D, Wang L. Health education and promotion at the site of an emergency: experience from the Chinese Wenchuan earthquake response. Global Health Promot 2016; 23(1): 15-26. 